\title{
兔疫学
}

\section{利用标记氨基酸参入法探讨 抗体对体外合成抗体的作用}

\author{
黄永安黃嘉陵 \\ （中国科学院实验生物研究所）
}

抗体对体內的免疫反应有一定的影响. Mason 等 ${ }^{[1]}, \mathrm{Uhr}$ 和 Baumann ${ }^{[2]}$ 和 Neiders 等 ${ }^{[3]}$ 认为抗毒素和溶血素均能抑制这些抗体的形 成，但也有实验证明其促进作用 ${ }^{[4]}$. 这说明 体內研究此问题的复杂性, 因实验是在抗原 存在的情况下进行的, 无法估计起实际作用 的部分. 我们利用了 $\mathrm{C}^{14}$ 一甘氨酸参入法来探 讨抗体对体外合成抗体的作用.

以结晶的牛血清白蛋白 (BSA) 靜脉高度 免疫家愁. 回忆刺激后, 颈动脉放血, 取出 脾脏. 以 $10^{7}$ 细胞/毫升的密度在 Eagle 溶液 加 $20 \%$ 的灭活免血清（正常血清或抗血清） 制成细胞悬液。每毫升悬液加进 1.5-2.0 微 居里的 $\mathrm{C}^{14}$ 一甘氨酸 (比放射性为 6.0 毫居里/ $\mathrm{mM}$ ). 于旋转管在 $37^{\circ} \mathrm{C}$ 温育 24 小时. 温. 育后，低温离心分出含有标记抗体和蛋白质 的上清液, 其放射性的计算均为 2-3 管培 液的平均数。 0 小时的样品为加入 $\mathrm{C}^{14}$ 一甘氨 酸后立郎分离出的上清液，作为对照.

实验组是用抗血清 (自体或异体)代替正 常血清加入 Eagle 溶液作为培液或正常血清 加从抗血清提出的丙种球蛋白 (1 毫克/毫 升). 对照组则含正常血清或正常血清加从
、正常血清提出的丙种球蛋白(同量).

总蛋白质的分离是以 TCA 沉淀, 再于 TCA $(5 \%) 95^{\circ} \mathrm{C}$ 处理 20 分钟, $95 \%$ 乙醇 $45^{\circ} \mathrm{C}$ 加热 10 分钟, 最后用乙醚洗涤, 得 蛋白沉淀. 分离特异性抗体是采用 “共同沉 淀” 法. 以最适量的抗血清和抗原溶液加入 培液, $37^{\circ} \mathrm{C}$ 保温后再搁置冰箱 $5-7$ 天。沉 淀于低温洗涤 3 次, 得带标记抗体的抗 原一 抗体络合物. 为了使分离前培液中的血清或 丙种球蛋白的浓度相同, 以免影响沉淀反 应，我们朵用了“交叉加入”法. 抗体和蛋白 沉淀均溶于 $\mathrm{NH}_{4} \mathrm{OH}(0.5 \mathrm{~N})$. 样品烘干后于 钟瞦盖革计数器测放射性. 抗体和蛋白均按 每毫升培液每分钟脉冲数计放射性.

从表 1 所列的结果可以看出, 与正常血 清组相比, 含自体抗血清的培液中一般可分 离出放射性较高的抗体 (和蛋白质). 为了阐 明此参入的增高是由于抗体本身的作用或是 与血清有关，进一步的实验说明异体的抗血 清的作用很不规律，有增加抗体的放射性 的，也有減低的. 同一抗血清对不同材料的

* 江子卿同志参加部分实验工作. 
表 1 含不同成分的培养液中的抗体和蛋白质的放射性

\begin{tabular}{|c|c|c|c|c|c|c|c|}
\hline \multirow{2}{*}{ 实验动物号 } & \multicolumn{7}{|c|}{ 放 射 性 (脉冲/分钟/毫升培液) ${ }^{1)}$} \\
\hline & & 正常血清 & 自体抗血清 & 异体抗血清 & 正 $r$-球 ${ }^{2}$ & 抗 $\gamma$-球 ${ }^{3)}$ & “纯”抗体" \\
\hline \multirow[t]{4}{*}{502} & 抗体 & 297 & 385 & $10(481)^{5)}$ & & & \\
\hline & & & & $23(491)$ & & & \\
\hline & 蛋白 & 2172 & 2380 & $122(481)$ & & & \\
\hline & & & & $107(491)$ & & & \\
\hline \multirow[t]{3}{*}{520} & 抗体 & 3303 & 4712 & $4113(483)$ & & & \\
\hline & 蛋白 & 7167 & 9097 & $92(466)$ & & & \\
\hline & & & & $9185(483)$ & & & \\
\hline \multirow[t]{2}{*}{540} & 抗体 & 1508 & 1331 & & 1503 & 1533 & \\
\hline & 蛋白 & 4662 & 4088 & & 5060 & 4960 & \\
\hline \multirow[t]{2}{*}{546} & 抗体 & 87 & 145 & & 64 & 114 & \\
\hline & 蛋白 & 380 & 667 & & 383 & 517 & \\
\hline \multirow[t]{2}{*}{551} & 抗体 & 3013 & 3988 & 853 (混合) & & & \\
\hline & 蛋白 & 6800 & 11738 & 1982(混合) & & & \\
\hline \multirow[t]{2}{*}{566} & 抗体 & 2993 & 3456 & $5123(551)$ & & & \\
\hline & 蛋白 & 12968 & 12473 & $16658(551)$ & & & \\
\hline \multirow[t]{2}{*}{568} & 抗体 & 2053 & 1638 & $1898(551)$ & & & \\
\hline & 蛋白 & 8612 & 6005 & $6148(551)$ & & & \\
\hline \multirow[t]{2}{*}{569} & 抗体 & 193 & 260 & $282(551)$ & & & \\
\hline & 蛋白 & 1597 & 1895 & $1742(551)$ & & & \\
\hline \multirow[t]{2}{*}{632} & 抗体 & 1220 & & & 1077 & 1275 & 1276 \\
\hline & 蛋白 & 2458 & & & 2363 & 2443 & 2762 \\
\hline \multirow[t]{2}{*}{645} & 抗体 & 3051 & 3256 & $2983(644)$ & & & \\
\hline & 蛋白 & 4403 & 4672 & $4017(644)$ & & & \\
\hline \multirow[t]{2}{*}{657} & 抗体 & 1603 & & & 1531 & 1505 & 1647 \\
\hline & 蛋白 & 3192 & & & 3040 & 3085 & 3040 \\
\hline
\end{tabular}

1) 溫育 24 小时的培液;

2) 正常血清的丙种球蛋白;

3）抗血清的丙种球蛋白;

4) 按 Sternberger 和 Pressman 法提取的抗体;

5）供抗血清的动物号.

影响也有变化, 而此变化则与血清的抗体血 凝效价无关. 如果在标准培液中 (Eagle 溶液 加 $20 \%$ 混合正常免血清)加进正常血清或抗 血清提取的包括抗体在內的丙种球蛋白，结 果指出，两组的抗体和蛋白的放射性均无显 著的差別. 同样的，“纯” 的抗体*也未能大 量提高标记甘氨酸的参入．以上的结果证实 了抗体分子对体外形成抗体沒有明显的作 用，而同时也说明了血清因系的复杂性. 自 体抗血清组的抗体和蛋白的增高很可能是因 为所用的血清是自体的，更有利于细胞的代
谢和蛋白合成的功能，而与抗体本身无关.

此外，表 1 还指出各实验的参入程度不 同，说明免疫动物对抗原的反应不一致而脾 脏细胞群体里具有产生抗体机能的细胞数变 化也大. 因此，抗体/总蛋白放射性的比例， 差別也较大.

从上述的结果和我们前一工作 ${ }^{[6]}$ 的报导 看来，体內高度免疫后的淋巴样细胞在体外 存活期间已具有一定形成抗体的能力，一切

* 按 Sternberger 和 Pressman ${ }^{[.5]}$ 法提取, 抗体为郑 一守同志供给。 
试图增強其功能的外加因素均无显著的作 用. 固然在目前的实验条件下，体內和体外 免疫反应的研究还存在着较大的差別, 但抗 体在体內也有可能不是直接作用于免疫活性 细胞.

\section{参考文献}

[1] Mason, J. H., Robinson, M. and Christensen,
P. A., J. Hyg., 53, 172-179 (1955).

[2] Uhr, J. W. and Baumann, J. B., J. Exp. Med., 113, 935-958 (1961).

[ 3 ] Neiders, M. E., Rowley, D. A. and Fitch, F. W., J. Immunol., 88, 718-724 (1962).

[4] Campbell, D. H., Am. J. Med., 15, 412 (1953).

[5] Sternberger, L. A. and Pressman, D., J. Immunol., 65, 65-73 (1950).

[6] 黄永安、葛锡锐, 实验生物学报, 8, 323-340 (1963).

\title{
微生物学
}

\section{胸腺中产生抗体的研究}

\author{
楊貴貞 赵裕琳 ${ }^{*}$ 馮家兴 ${ }^{* *}$ 胡明杰 $^{* * *}$ \\ 赵 琳 ${ }^{* * * *}$ 张紹伦 彭大才 \\ （吉林医科大学微生物教㸴室）
}

胸腺对机体免疫生成的意义，1960年以 后才为人们所重视. 现今大多数免疫学工作 者都认为，胸腺控制机体一切免疫反应. 据 此, 可以推测胸腺对抗体产生能起到一定作 用. 至于胸腺组织能否直接产生抗体, 至今 晌是个未能最后解决的问题。根据文献 报 导 $^{[1-5]}$; 一般认为胸腺组织本身是不能产生 抗体的. 为了澄清这一问题, 本文进行了实 验观察。

本文用大鼠以白喉类毒素进行免疫，观 察胸腺中抗体产生及细胞反应。

大鼠体重为 200 克左右, 年龄为 $2-3$ 个月. 分 6 组,每组 6 只. 均于两侧后脚掌注
入白喉类毒素各 0.2 毫升 (每毫升为 $40 \mathrm{Lf}$ ), 共 0.4 毫升，间隔 2 周，再注入同样数量的 抗原. 于二次免疫后 $5 \frac{1}{2}$ 个月进行再次免 疫. 第一组为再次免疫前对照组, 第二、 三、四、五组分別为再次免疫后 4、7、10 及 14 天解剖者. 第六组为正常对照组.

由心脏取血, 杀死动物, 取材观察. 取 腘窝淋巴结及脾脏, 接着开胸腔, 取胸腺,

\footnotetext{
* 新疆筑学院.

** 贵阳医学院.

****湖南医学院.

**** 北京中医学院。
} 